those drugs which are included in the British National Formulary would be acceptable to the majority, and would still give each individual doctor the right to prescribe as he felt best in special situations. Furthermore, such limitation would actually improve the overall standard of prescribing especially if the advice given in the first half of the B.N.F. was observed. The present machinery, whereby the cost of a doctor's prescribing is kept under review and he is "contacted" if it becomes excessive, could be modified to pay special attention to the non-B.N.F. component as well as to the total cost. In 19723,035 "contacts" were made by regional medical officers as regards doctors prescribing. Only on one occasion was further action taken and eventually a small deduction was made from the doctor's remuneration because of excessive prescribing. In 19732,987 contacts were made but it appears that no further action was taken. I cannot believe that this one case was the only example of persistent overprescribing in two years and I feel that, to be effective, action must be taken far more frequently.

A few years ago two expert panels assessed all the drugs in M.I.M.S. and came to the conclusion that $35 \%$ of the preparations listed were undesirable. While the situation may have improved since, perhaps the time has now come for the D.H.S.S. to refuse to accept financial responsibility for the cost of prescriptions for preparations which are widely held to be undesirable (subject, of course, to reasonable appeal). Such a course would be infinitely preferable to a selective list of prescribable drugs, which would seriously affect the pharmaceutical industry. A strong and reasonably profitable industry is desirable both for future research and also to maintain exports (trade surplus-exports over imports-in 1973 was $£ 154$ million).

\section{Economy and Efficiency}

It is difficult to assess how much money would be saved by these measures, but they should tend to improve the overall standard of prescribing. Certainly far greater economy could be achieved if doctors were more critical of their prescribing and reassessed the need for each prescription carefully instead of just ordering a "repeat" when the patient reattends. (Evidence for overprescribing includes the results of collections of unwanted drugs -1 tons were collected a few years ago in one week in S. Wales.) Too often a p.r.n. prescription for night sedation is given as part of the hospital admission procedure, thus initiating an undesirable addiction. Bronchitics with irreversible airways obstruction frequently receive a useless prescription for bronchodilators when forceful advice about smoking would be more appropriate. Sore throats are often due to a viral infection but, when antibiotics are considered necessary, relatively expensive drugs, such as ampicillin, are often prescribed in place of the therapeutically more desirable drug of choice phenoxymethylpenicillin. Numerous other examples could be mentioned especially in relation to tranquillizers, antidepressants, antirheumatics, and antibiotics where a doctor often appears to be biased towards a particular preparation as a result of advertising rather than consideration of the results of scientific and clinical evaluation.

To conclude I propose that doctors be asked to voluntarily limit their prescribing to drugs recommended in the British National Formulary and that sanctions be more vigorously applied to restrain those who, in the view of their colleagues, regularly overprescribe. If we do not organize this inside the profession, more stringent restrictions may eventually be imposed from outside.

NOEL D. L. OLSEN, M.B., M.R.C.P., Medical Registrar

\section{Do All our Clinicopathological Investigations Pay Off?}

\section{N. K. Shinton}

Like most procedures in medicine, financial evaluation or costeffectiveness of clinicopathological investigations is almost impossible owing to variables such as time saved in hospital beds, time off work by patients, or psychological benefit to the patient-all of which may be influenced by factors other than the results of laboratory investigations. Hence the economic assessment of laboratory procedures is limited to studying costs of staff and equipment in relation to work load. Unfortunately the methods at present employed by the D.H.S.S. for correlating work load with staff are based on the number of requests and tests without any consideration of the enormous differences in the time and technical experience required from one test to another. In haematology the test time may vary from one minute for an erythrocyte sedimentation rate to 120 minutes for a red-cell enzyme assay; manual procedures taking nine minutes can be processed with print-out of results by an automated machine in one. A system of unit values for each test therefore has much to commend it.

Excluding capital development, present laboratory running costs are $80 \%$ for salaries and $20 \%$ for maintenance and replacement of equipment. So if there are to be effective economies in the laboratory service of the N.H.S. the staff/work load ratio must be improved by increasing the availability of equipment able to handle large work loads. Unfortunately the N.H.S. has a long way to go: about 500 laboratories are carrying out haematological tests but 300 of these perform less than 30,000 requests per annum (120 per day). Thus in most haematology laboratories expensive automated equipment would not be cost-effective. On the other hand, many of these laboratories are necessary to cover essential emergency procedures.

\section{Rationalization}

The staff/work load ratio could be improved for manual procedures by limiting the number of laboratories where each test is performed. For many procedures it takes little longer to perform 10 tests than a single one, and this applies similarly to clinical chemistry and to a lesser extent to microbiology. If N.H.S. finances are to be restricted the policy that every district general hospital should have a laboratory offering a complete range of tests cannot be maintained. There are two alternatives: to continue with these laboratories-limiting the number, range, and quality of the tests-or to rationalize the work among the various N.H.S. laboratories. Asking clinicians faced with a rising population of patients to reduce the load on the laboratory is not possible, and reduction in quality means wasted effort. Hence a reduction in the range of routine tests offered by each laboratory is the only solution.

The D.H.S.S. has made a start in rationalization by setting up supraregional laboratories for some procedures. Rationalization is unpopular with both laboratory and clinical staff because inevitably it leads to lack of personal communication, delay in transport of specimens and return of reports, and a lessened attractiveness of a laboratory for training. To some extent these drawbacks can be avoided by preliminary explanation, improved transport, the installation of data processing, and integration of training programmes among laboratories. Unless N.H.S. laboratories become more efficient economically it is unlikely that money will be available in the near future for new developments-and without these interest in medicine will decline and standards of practice fall.

\section{Department of Haematology, Coventry and Warwickshire Hospital,} Coventry CV1 4FH

N. K. SHINTON, M.R.C.PATH., F.R.C.P., Consultant Haematologist

\section{Can We Audit Cost-effectively?}

\section{Hugh Dudley}

I am worried both about the title and the implications of my subject. The title suggests the excessively transatlantic jargon of the economist, though .I am relieved to see in the Oxford English Dictionary that one of the first uses of audit was more general, as a "searching examination or solemn rendering of accounts especially the Day of Judgement". Cost-effective is a Johnny-come-lately but has reached the O.E.D. Supplement, probably as a result of Mr. McNamara's stay at the Pentagon in the mid-sixties. If I were patient enough to search through the economic literature I could probably find it well defined, but for me it means that if we spend a penny on analysing what we do we get a penny or more back in the good that accrues to the community. And there's the rub, because, if Mr. McNamara's analysis of the cost of the F 1-11 fighter-bomber versus its strike 